spatial resolution, since angles $\theta$ of a few degrees at most can be employed, in 1978 they developed a technique whereby two laser beams intersected in a small plasma volume. Narrow-angle scattering was observed from each beam, but by using a cross-correlation technique only fluctuations common to both were recorded.

BIBLIOGRAPHY

1. Sheffield J., Plasma Scattering of Elec tromagnetic Radiation (Academic Press) 1976.
2. Morgan P.D., Green M.R., Siegrist M.R. and Watterson R.L., "The development of far-infrared lasers for Thomson-scattering measurements on tokamak plasmas", Comments Plas. Phys. Cont. Fusion 5 (1979) 4, p. 141

3. Forrest M.J., Carolan P.G. and Peacock N.J. "Measurement of magnetic fields in a tokamak using laser light scattering", Nature 271 (1978) 5647, p. 718.

4. Surko C.M. and Slusher R.E. " "Study of plasma density fluctuations by the correlation of crossed $\mathrm{CO}_{2}$ laser beams", Phys. Fluids 23 (1980) 12, p. 2425.

\section{Applications to Biology and Medicine}

\section{A. Andreoni, R. Cubeddu and C.A. Sacchi, Milan}

(Quantum Electronics and Electronic Instrumentation Centre of the CNR)

Ever since it first appeared, the laser has been considered a highly promising instrument for biomedical research. As early as 1962, Britton Chance (Johnson Research Foundation) reported, at the annual meeting of the American Biophysical Society, on a study of electron transfer in photosynthetic algae, made with a ruby laser. In the same year, M. Bessis (Ecole Pratique des Hautes Etudes, Paris) published his results on "Irradiation des organites cellulaires à l'aide d'un laser à rubis", and C.J. Campbell (Columbia University, N.Y.) published a paper on the use of "The optical maser as a retinal coagulator".

It will be recalled that the annual conference of the Quantum Electronics Division of EPS, held in Florence in 1979, was devoted to "Lasers in Photomedicine and Photobiology".

Applications of the laser to Biology and Medicine are diverse, exploiting one or more of the special properties of the device and the measuring techniques that have been described in relation to chemistry and other disciplines. But besides being a diagnostic tool, a laser can be used to act on a biological sample. Examples of such applications are the laser activation of photosynthesis, the micro-irradiation of cells and cellular organelles, and, in medicine, the photodynamic action of some drugs, surgery, and photocoagulation.

\section{Biology}

Many structural and conformational properties of the most relevant biomolecules (DNA, RNA, proteins, lipids, etc.) have been studied "in vivo" by means of laser spectroscopy, notably emission spectroscopy performed on suitable dyes that label these biomolecules. In particular, time-resolved fluorescence and polarization have been applied successfully. This is due to the fact that the binding of a dye molecule to a biomolecule induces marked changes in certain fluorescence properties such as the non-radiative decay pathways of its excited state.

Studies on DNA have been made with Acridine dyes which exploit this effect. The DNA-specific staining dyes Proflavine and Quinacrine Mustard (OM) were chosen, because their fluorescence properties depend on the DNA site where they bind. In particular, they intercalate between adjacent base-pairs in the DNA double helix, and have a longer excited-state ( $S_{1}$ ) lifetime when they intercalate two AdenineThymine (AT) base-pairs than when they are bound to sites containing at least one Guanine-Cytosine (GC) base-pair. Although the origins of this property were unknown, the DNA base-pair composition of a number of substances could be determined.

It then proved possible to explain the reasons for the behaviour: these molecules undergo excited-state proton transfer and the rates of proton exchange are influenced by the base-pair composition of the DNA binding site.

Many other experiments have been made in which the same dyes have been used to probe some structural property of the DNA "in vivo", including some on a microscopic scale using a microfluorometer. In this, the exciting laser pulse (generated by a nitrogen-pumped tunable dye laser) is concentrated through a microscope objective down to a spot of $0.5 \mu \mathrm{m}$ diameter; the subsequent fluorescence is detected by a fast photomultiplier ( $0.2 \mathrm{~ns}$ risetime). Among the applications of this instrument, can be mentioned a study of the DNA base-pair composition in single bacteria with $\mathrm{QM}$.

With the same instrument also, experiments in fluorescent-drug microdosimetry, such as the study of cell or tissue uptake of antitumour drugs) can be made. In this, the initial strength of the fluorescence pulse is related to the local amount of drug, while its time evolution is indicative of the physical condition of the drug molecule.
Fluorescent labels find another important application in flow cytometry. In this technique, mammalian cells in suspension are lined up by passing through a special flow chamber, and exposed one at a time to a laser beam. Crossing the beam, each cell produces one or more optical pulses that are related to its DNA (or RNA) content, dimensions and so on. This may allow discrimination between tumour and normal cells, and analysis of the cell cycle or drug effects. It is the only way of making rapid quantitative measurements on large numbers of cells (typically, at a rate of $5 \times 10^{4}$ cells $/ \mathrm{min}$ ), with high statistical precision. Commercial versions of the instrument have been on the market for several years, and are currently used in several hospitals and institutes of biomedical research.

Very recently, we have conducted new experiments, in which Acridines were used as intermediates to act on the DNA, rather than to probe it. The effect is produced via a two-step photoionization of the dye molecule, and results in damage to the DNA base pairs that it intercalates. Different schemes for the interaction of intense laser pulses have been proposed, in order to obtain an overall efficiency of the photoionization process that is dependent on the $S_{1}$ lifetime. The irradiation of $\mathrm{QM}$, bound to synthetic polynucleotides containing only GC or AT bases, with nitrogen laser pulses of length shorter than the $\mathrm{S}$, lifetime of $\mathrm{QM}$ bound to $\mathrm{AT}$, but longer than that of $\mathrm{QM}$ bound to $\mathrm{GC}$, resulted in a destruction of the complex $\mathrm{QM}: \mathrm{GC}$ one fifth that of the complex QM:AT. A much higher contrast (a factor of over 40 ) between the photodamage of the two complexes was obtained using Proflavine (PF) as the staining agent and pulses from two lasers of different wavelengths with a delay between them.

\section{Medical Research}

The laser began to figure in medical research at an early stage, the first application being coagulation in ophthalmology using a ruby laser. This is now done with an argon laser, and is accepted throughout the world as the treatment for retinal detachment and for nonproliferative retinopathy.

After the invention of the $\mathrm{CO}_{2}$ laser, the surgical applications began to increase rapidly. When focused on the surface of any living tissue, the $\mathrm{CO}_{2}$ laser beam is strongly absorbed (in a layer of $\sim 200 \mu \mathrm{m}$ ) by the water contained in the tissue $(80-90 \%)$, and transformed into heat. With an intensity of a few $\mathrm{W} / \mathrm{mm}^{2}$, the tissue fluids boil almost instanteneously, and the tissue vaporizes. If the focal point of the beam is moved over the tissue, an incision results of a depth that depends on the intensity of the beam and on the speed of the movement. The incision is relatively blood- 
less, at least if vessels with a diameter smaller than $1 \mathrm{~mm}$ are transected, and this can be a definite advantage.

The $\mathrm{CO}_{2}$ laser, coupled to an operating microscope equipped with a micromanipulator for beam deflection, has found important applications in microsurgery. In the field of ear, nose and throat surgery for example, $\mathrm{CO}_{2}$ laser microsurgery provides precise and completely haemostatic incision in the larynx and makes it possible to carry out procedures that could otherwise hardly be performed endoscopically. Similar claims can be advanced in gynaecology and neurosurgery. $\mathrm{CO}_{2}$ laser microsurgery is now in use all over the world and applications are growing all the time.

Two other lasers, the argon-ion and the Nd-YAG, have also found important applications in medicine. They are used to stop haemorrhages from various lesions in the gastrointestinal tract. Argon laser radiation is well absorbed by haemoglobin, while Nd-YAG radiation penetrates deeply into the gastrointestinal mucosa. These properties make the former more suitable for superficial lesions and the latter for massive haemorrhages. Optical fibres (usually of quarz) are used to transmit the laser beam via one of the channels of a flexible gastroscope. In contrast to other techniques (electrocoagulation, cryo- or heaterprobes), laser coagulation allows the energy to be applied without contact and gives better control of tissue necrosis.

A new field of application that uses lowintensity visible lasers is based on the interaction of light with fluorescent drugs, in particular haematoporphyrin-derivative, for the localization and treatment of several types of tumour. This technique is based on the higher accumulation (and/or retention) of the drugs in malignant tissues than in most normal tissues. Thus, proper illumination can cause the tumour area to show up as the fluorescence of the dyes makes them easily visible.

There is also a potential therapeutic effect related to their photodynamic action: the destruction of the tissue where they reside after activation with visible light in the presence of oxygen. The process occurs through energy transfer from the excited dye molecule to endogenous oxygen, with formation of the cytotoxic agent "singlet oxygen".

Several research groups all over the world are at the moment deeply involved in defining the effect of haematoporphyrin and light in cancer therapy. The activation light at :he wavelength of $630 \mathrm{~nm}$ is provided usually by a cw rhodamine $B$ dye laser pumped by an argon laser. This laser is a very useful tool for two main reasons:

(i) it provides the $\mathrm{cw}$ high power needed to treat tumour areas (the average power required is $\left.100-200 \mathrm{~mW} / \mathrm{cm}^{2}\right)$;

(ii) via optical fibres it is possible to reach tumours inside the body (as for example in

\section{Optica Acta}

\section{A Europhysics Journal published by Taylor \& Francis Ltd in collaboration with The Institute of Physics}

An international journal of modern optics and laser physics covering most experimental and theoretical aspects of optics and their applications, over spectral regions extending from $x$-rays to the infrared, in fields such as quantum optics, optical materials, electro-optics, fibre optics, diffraction and interference, free and guided propagation, scattering, optical coherence, photon statistics, holography and speckle, lasers, nonlinear optics, integrated optics, image formation, image processing, polarisation, thin films and multilayer coatings, physiological optics, colorimetry, optical metrology, optical instrumentation and techniques, optical design, optical production technology and optical system testing.

For a specimen copy and further information, please write to:

Taylor \& Francis Ltd

4 John Street

London WC1N 2ET

the lung or in the bladder) or to deliver light directly to tumour masses.

Many human patients $1 \sim 400$ at the Roswell Park Memorial Institute, Buffalo, N.Y., by T.J. Dougherty) and animals have so far been treated, and the response obtained seems to be very encouraging. In most cases, in fact, the disappearance or a reduction of the tumour has been observed after the treatment. Photochemotherapy thus seems to provide a new promising treatment that may, in some cases, be complementary to current cancer therapies.

In conclusion, we believe that the laser has already made a great contribution to the progress of biomedical research and, in some cases, to current clinical applications. This contribution will certainly increase with a better understanding of the basic photobiologic effects.

\section{REFERENCES}

The interested reader is referred to:

1. "Lasers in Photomedicine and Photobiology", Proc. EPS Quantum Electronics Division Conf., Florence, Italy, Sept. 3-6, 1979, ed. by R. Pratesi and C.A. Sacchi (Springer-Verlag) 1980.

2. "Lasers in Biology and Medicine", Proc. NATO Advanced Study Institute, Camaiore, Ita1y, Aug. 19-31, 1979, ed. by F. Hillenkamp, R. Pratesi and C.A. Sacchi (Plenum Press) 1980.

\section{First European Conference of the Atomic Physics Division}

The first European Conference on Atomic Physics (ECAP) was held in Heidelberg, 6-10 April 1981. The essential idea of this conference was to combine the different annual national meetings in the fields of atomic and molecular physics, into a European conference and, in this way, bring together scientists from all European countries. The scientific scope of the conference was representative of the four Sections of the Atomic Physics Division of EPS: Atomic Spectroscopy, Electronic and Atomic Collisions, Molecular Physics and Chemical Physics. With 900 participants, 33 invited speakers and over 510 contributed papers this first ECAP was a real success, particularly due to the excellent organisation by the Chairman of the conference, Professor zu Putlitz, who supported the idea of a European Conference with strong personal initiative and energy.

It is not possible and also not the intention to review the whole scientific scope of the Heidelberg Conference; rather I shall report on a few invited papers which I believe to be important examples from among the variety of problems currently being investigated in atomic and molecular physics.

In his talk on line widths and line shapes in molecular photoionization, Ch. Jungen showed in a clear and illustrative manner, how effectively multichannel quantum defect theory (MODT) can be applied to 